\title{
Human Brain Pyridoxal-5'-phosphate Phosphatase: Production and Characterization of Monoclonal Antibodies
}

\author{
Dae Won Kim ${ }^{\ddagger}$ Won Sik Eum ${ }^{\ddagger}$ Hee Soon Choi, So Young Kim, Jae Jin An, Sun Hwa Lee, \\ Eun Joung Sohn, Seok-Il Hwang, Oh-Shin Kwon ${ }^{\dagger}$, Tae-Cheon Kang*, Moo Ho Won;, \\ Sung-Woo Cho ${ }^{\S}$, Kil Soo Lee, Jinseu Park* and Soo Young Choi* \\ Department of Biomedical Science and Research Institute for Bioscience and Biotechnology, Hallym University, \\ Chunchon 200-702, Korea \\ 'Department of Biochemistry, Kyungpook National University, Taegu 702-701, Korea \\ Department of Anatomy, College of Medicine, Hallym University, Chunchon 200-702, Korea \\ s Department of Biochemistry and Molecular Biology, University of Ulsan College of Medicine, Seoul 138-736, Korea.
}

Received 15 July 2005, Accepted 14 September

\begin{abstract}
We cloned and expressed human pyridoxal-5'-phosphate (PLP) phosphatase, the coenzymatically active form of vitamin $B_{6}$, in Escherichia coli using pET15b vector. Monoclonal antibodies (mAb) were generated against purified human brain PLP phosphatase in mice, and four antibodies recognizing different epitopes were obtained, one of which inhibited PLP phosphatase. The binding affinities of these four mAbs to PLP phosphatase, as determined using biosensor technology, showed that they had similar binding affinities. Using the anti-PLP phosphatase antibodies as probes, we investigated their cross-reactivities in various mammalian and human tissues and cell lines. The immunoreactive bands obtained on Western blots had molecular masses of $c a .33 \mathrm{kDa}$. Similarly fractionated extracts of several mammalian cell lines all produced a single band of molecular mass $33 \mathrm{kDa}$. We believe that these PLP phosphatase mAbs could be used as valuable immunodiagnostic reagents for the detection, identification, and characterization of various neurological diseases related to vitamin $B_{6}$ abnormalities.
\end{abstract}

Keywords: Crossreactivity, Epitope mapping, Human PLP phosphatase, Immunoblot, Monoclonal antibodies

\footnotetext{
${ }^{\ddagger}$ These first two authors contributed equally to this work

*To whom correspondence should be addressed.

Tel: 82-33-248-2112, Fax: 82-33-241-1463

E-mail: sychoi@hallym.ac.kr and jinpark@hallym.ac.kr
}

\section{Introduction}

Pyridoxal-5'-phosphate (PLP) is the coenzymatically active form of vitamin $\mathrm{B}_{6}$ and plays an important role in maintaining the biochemical homeostasis (Meister, 1990; Snell, 1990). Thus, the other nutritionally available vitamer forms in this family must be converted to PLP. The enzymes conventionally involved in this metabolism are an ATP-dependent pyridoxal kinase and a flavin mononucleotide-dependent pyridoxine-5'phosphate (PNP) oxidase (McCormick et al., 1961; Kwok and Churchich, 1992; Hanna et al., 1997; Ngo et al., 1998).

The major pathways of vitamin $\mathrm{B}_{6}$ metabolism were established decades ago, but little is known about how the concentration of PLP is controlled in mammalian tissues. Studies on PLP homeostasis have shown that several mechanisms are involved. The factors involved in the regulation of PLP may include catabolism of PLP by PLP phosphatase, pyridoxal kinase, and PNP oxidase, the degree of protein binding of synthesized coenzyme, and precursor transport (Snell and Haskell, 1971; Anderson et al., 1974). Although PNP oxidase plays role a kinetic role in the regulation of PLP formation (Kwon et al., 1991), PLP availability is mainly dependant on protein binding and phosphatase action (Snell and Haskell, 1971; Li et al., 1974; Lumeng and Li, 1975).

A PLP specific phosphatase catalyzes the dephosphorylation of PLP, and PNP and has been purified from human erythrocytes (Fonda, 1992). Molecular properties, including molecular weight, optimum $\mathrm{pH}$, substrate specificity, and metal requirements, indicate that PLP phosphatase differs from other known phosphatases. Alkaline phosphatase (EC 3.1.3.1) and acid phosphatase (EC 3.1.3.2) also hydrolyze PLP and pyridoxamine 5'-phosphate (PMP), but have broad substrate specificities for phosphomonoesters (Harris, 1990; Bull et al., 2002). Alkaline 
phosphatase is resistant to fluoride and is inhibited by $\mathrm{L}(+)-$ tartrate and levamisole, whereas PLP specific phosphatase is not. Moreover, acid phosphatase is not inhibited by fluoride and EDTA. The PLP phosphatase is most active at physiological $\mathrm{pH}$ 's, and requires $\mathrm{Mg}^{2+}$ for activity (Fonda and Zhang, 1995). It is a dimer of molecular mass $64 \mathrm{kDa}$, and has a $K m$ value of approximately $1 \mathrm{mM}$ for PLP, which is 10-fold lower than that reported with other phosphatases (Fonda, 1992). Data obtained by chemical modification studies reveal that PLP phosphatase possess essential residues, namely, cysteine, arginine, histidine, and a carboxylate group at or near its active site (Gao and Fonda, 1994a; 1994b; 1994c). We previously reported on the cloning and characterization of a human and mouse brain PLP phosphatase (Jang et al., 2003). Moreover, the cDNA sequence of mouse PLP phosphatase showed a high degree of (95\%) similarity with human PLP phosphatase.

PLP phosphatase is of particular interest because of the intimate relationship between vitamin $\mathrm{B}_{6}$ metabolism and neurological disorders. Several lines of evidence indicate that convulsive seizures occur during vitamin $\mathrm{B}_{6}$ deficiency (Gospe et al., 1994; Glenn et al., 1995; Waymire et al., 1995). Despite the essential roles of PLP phosphatase in various vitamin $\mathrm{B}_{6}$-related disorders, structural and regulatory studies are incomplete. Therefore, in the present study, the expressed human PLP phosphatase was injected as an immunogen into BALB/c mice to obtain monoclonal antibodies (mAbs), and four $\mathrm{mAbs}$ to the protein were produced by fusion experiments. Those that recognized PLP phosphatase on Western blots were characterized and used as probes in a cross-reactivity study on PLP phosphatase from humans and other mammals and from chicken.

\section{Materials and Methods}

Materials Restriction endonuclease, T4 DNA ligase, and the human brain cDNA library were purchased from Takara. Plasmid pET15b, E. coli BL21 (DE3) and the $\mathrm{Ni}^{2+}$-affinity column were from Novagen. Basal medium, Dulbecco's Modified Eagle's (DME) medium, hypoxanthine-aminopterin-thymidine (HAT), fetal bovine serum (FBS), and penicillin/streptomycin antibiotics were purchased from Gibco-BRL, and goat anti-mouse IgG conjugated with alkaline phosphatase (AP) was from Santa Cruz Biotechnology, Inc. Hybond- $\mathrm{C}^{+}$nitrocellulose membranes and ECL Western blotting detection reagent were from Amersham Pharmacia Biotech Inc. All other reagents were of the highest purity obtainable.

Enzymatic assay of PLP phosphatase The purification and enzyme assays of PLP phosphatase were performed as described by Jang et al. (2003). Briefly, the enzymatic activity of PLP phosphatase was measured at $\mathrm{pH} 7.4$ in $40 \mathrm{mM}$ triethanolamineamine- $\mathrm{HCl}$. The rate of production of pyridoxal from PLP was measured by following a reduction in absorbance at $390 \mathrm{~nm}$ for at least $3 \mathrm{~min}$. One unit of specific activity was defined as the amount of protein that catalyzed the formation of pyridoxal $/ \mathrm{min}$ from PLP (Jang et al., 2003).
Production of anti-human PLP phosphatase monoclonal antibodies The production of mAbs to human PLP phosphatase was performed as previously described (Choi et al., 1996; Eum et al., 2005). Briefly, the purified enzyme was mixed with an equal volume of complete Freund's adjuvant and was injected intraperitoneally into a BALB/c mouse (6-8 weeks old). Two booster injections with incomplete Freund's adjuvant at 3-week intervals were followed by a final injection without adjuvant at 3 days before the cell fusion experiment. Feeder layer cells were prepared 1 day before fusion. For fusion, prepared spleen cells and SP2/o-Ag-14 mouse myeloma cells were combined and collected in a $50 \mathrm{ml}$ disposable tube by centrifugation at $650 \mathrm{~g}$ for $5 \mathrm{~min}$. After removing the supernatant completely, the cell pellet was mixed by tapping the tube with fingers. Then, $1 \mathrm{ml}$ of 50\% PEG was added over a period of $1 \mathrm{~min}$ with constant swirling. The fusion reaction was stopped after 90 s by slowly adding $10 \mathrm{ml}$ of DMEM. Positive clones were first selected by immunodot-blot analysis and confirmed by Western blotting.

Immunoblot analysis For immunoblotting, small squares $(1 \times 1$ $\mathrm{cm})$ were drawn on a sheet of nitrocellulose paper $(10 \times 10 \mathrm{~cm})$ and marked by numbering. One microliter of antigen solution $(0.5 \mathrm{mg} /$ $\mathrm{ml}$ ) was then applied onto each square and air-dried (Lee et al., 2003). Blots were incubated for $1 \mathrm{~h}$ in Blotto [ $2 \%$ nonfat dry milk in Tris-buffered saline (TBS)], rinsed briefly with TBS, and airdried. The blots were then processed using the Western blotting procedures described by Towbin et al. (1996) using the produced PLP phosphatase mAbs.

Purification monoclonal antibodies To purify the monoclonal antibodies, $50 \mathrm{ml}$ of culture supernatant was clarified of cells and insoluble aggregates by centrifuging for $30 \mathrm{~min}$ at $15,000 \times \mathrm{g}$ and then applied to a $1 \mathrm{ml}$ protein A-agarose column (Sigma). The column was washed with PBS until the absorbance of unbound proteins reduced to the background level, and antibodies were eluted with $0.1 \mathrm{M}$ glycine- $\mathrm{HCl}$ ( $\mathrm{pH} 2.5$ ). The eluted antibodies were neutralized by adding $1 \mathrm{M}$ Tris ( $\mathrm{pH}$ 8.0) and dialyzed against PBS.

Cross-reactions between PLP phosphatase from different sources Human, bovine, rat, chicken, rabbit, cat, dog, and porcine brain tissues and several rat tissues (brain, heart, lung, liver, stomach, muscle) were removed and homogenized in $10 \mathrm{mM}$ potassium phosphate containing $0.1 \mathrm{mM}$ EDTA, $1 \mathrm{mM} \beta$-mercaptoethanol, and $1 \mathrm{mM}$ PMSF. Individual $25 \%(\mathrm{w} / \mathrm{v})$ homogenates were centrifuged at $10,000 \times g$ for $1 \mathrm{~h}$ and $30 \mu \mathrm{g}$ of each total protein was applied to SDS-PAGE and transferred to nitrocellulose membranes. The lysates of several mammalian cell lines, i.e., PC12, J774A1, HepG2, SiHa, and HL3T1 were separated by SDSPAGE and transferred to nitrocellulose membranes, and all blots were processed by Western blotting, as described above. In the case of human brain, total proteins were prepared by homogenizing a small fragment of cerebral cortex removed from a 45-year old male during surgery after an accident.

Enzyme inhibition test The inhibitory effects of mAbs on PLP phosphatase activity were investigated by measuring the catalytic activity of PLP phosphatase $(5 \mu \mathrm{g})$ after pre-incubation with purified $\mathrm{mAb}(50 \mathrm{mg})$ for $1 \mathrm{~h}$ at $37^{\circ} \mathrm{C}$. Enzymatic assays of the samples were measured using the enzyme assay method described above. 
Epitope mapping One-dimensional epitope mapping was carried out using a previously described procedure (Choi et al., 1995; 1996). Ten micrograms of purified human PLP phosphatase in SDS sample buffer were mixed with an equal volume of Staphylococcus Aureus V- 8 protease solution ( $0.3 \mu \mathrm{g}$ in SDS sample buffer). The mixtures were assayed using SDS polyacrylamide gel and separated peptides were transferred for immunoblot analysis, as described above.

Immobilization and analysis of enzyme-PLP phosphatase mAb interactions on BIAcore Protein-protein interactions between monoclonal antibodies and PLP phosphatase were examined using a Pharmacia Biosensor BIAcore instrument. CM5 research-grade sensor chips (Pharmacia Biosensor) were used for all experiments. The indirectly oriented immobilization of antibodies on CM5 sensor chips were carried out as follows. First, rabbit anti-mouse IgG Fc (ramfc) was coupled to a chip by injecting $100 \mathrm{ng}$ of ramfc in $10 \mathrm{mM}$ sodium acetate $(\mathrm{pH} 4.5)$ at a flow rate of $5 \mu \mathrm{l} / \mathrm{min}$ at $20^{\circ} \mathrm{C}$. The carboxyl-methyl dextran matrix of the sensor chip was activated by injecting $30 \mu \mathrm{l}$ a mixture of $0.2 \mathrm{M}$ 1-ethyl-3[(dimethylamino)propyl]carbodiimide and $0.05 \mathrm{M} \mathrm{N}$-hydroxysuccinimide in water for 6 min to convert the carboxyl groups of the sensor chip matrix to N-hydroxy-succinimide ester groups. This ester is susceptible to nucleophilic attack by the amino groups of proteins, which causes an amide linkage between the protein and the sensor chip. Under these conditions, typically 3,700 resonance units of ramfc were immobilized on a CM5 chip. The interactions between PLP phosphatase and monoclonal antibodies were measured by two subsequent injections; the monoclonal antibodies were captured by ramfc, and followed by PLP phosphatase. Proteinprotein interaction studies were carried out in HEPES-buffered saline (10 mM HEPES/KOH (pH 7.5), $150 \mathrm{mM} \mathrm{NaCl}, 3.4 \mathrm{mM}$ EDTA, and $0.005 \%$ surfactant P20). Kinetic rate constants $\left(k_{\text {off }}\right.$ and $\left.k_{\mathrm{on}}\right)$ and equilibrium dissociation constants $\left(K_{\mathrm{D}}=k_{\mathrm{off}} / k_{\mathrm{on}}\right)$ were determined using BIAlogue Kinetics Evaluation Software.

Other methods Discontinuous SDS-PAGE was carried out as described by Laemmli (1970). Spectrophotometric measurements were carried out using a Kontron model UVIKON 930 doublebeam spectrophotometer.

\section{Results and Discussion}

Expression and purification of human brain PLP phosphatase To produce human brain PLP phosphatase monoclonal antibodies, we cloned and expressed a recombinant fusion protein in bacteria. The $\mathrm{pET} 15 \mathrm{~b}$ bacterial expression vector used contained six consecutive histidine residues and an 891 bp PLP phosphatase gene at the amino-terminus of inserts to help purify fusion proteins. Briefly, the recombinant protein was expressed in E. coli BL21 (DE3) and purified using a $\mathrm{Ni}^{2+}$-nitrilotriacetic acid Sepharose column (Qiagen), according to the manufacturer's instructions. Salts in the purified protein were removed by PD10 column chromatography (Amersham, Sweden). The purified fusion protein obtained was nearly homogenous, as determined by SDS-PAGE, Coomassie Brilliant
(A)

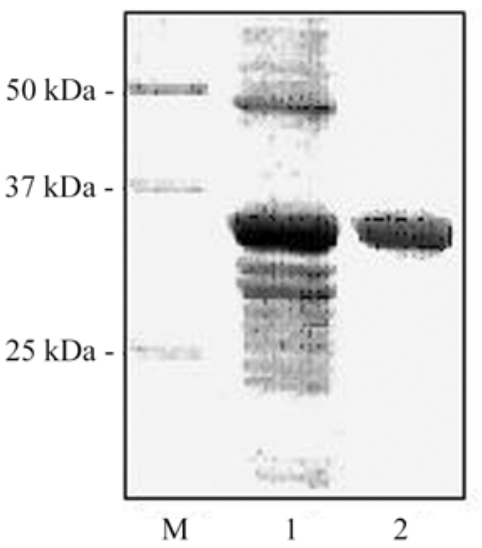

(B)

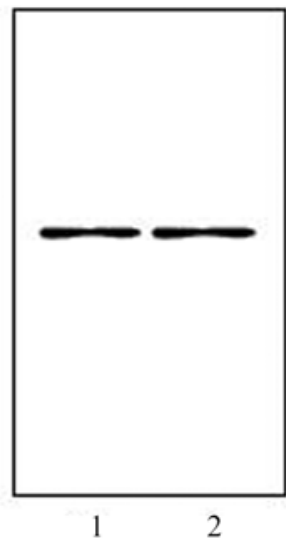

Fig. 1. Purification and Western blot analysis of human PLP phosphatase fusion protein. (A) Analysis of purified PLP phosphatase fusion proteins by Coomassie blue staining. PLP phosphatase fusion proteins were purified by affinity chromatography on a Nickel-Sepharose column, followed by gel filtration on a PD10 column. (B) Western blot analysis of the purified PLP phosphatase fusion proteins using polyclonal antihistidine antibody (Santa Cruz, 1: 500). Lanes in (A) and (B) are as follows: lane 1, induced; lane 2, purified.

blue staining, and by immunoblotting with polyclonal antihistidine antibody (Fig. 1). The purified protein had a specific activity of 1.3 units $/ \mathrm{mg}$ and appeared as a single band with a molecular mass of $33 \mathrm{kDa}$ on SDS-PAGE.

Production and characterization of monoclonal antibodies Human PLP phosphatase purified as described above by affinity chromatography, was used to generate monoclonal antibodies (mAbs). To enhance its immunogenicity and obtain antibodies with optimal reactivity on Western blots, the purified enzyme was denatured in SDS and injected into BALB/c mice. In two fusion experiments, 79 positive clones were initially screened by immunodot-blot analysis, because goat anti-mouse IgG antibody was used as a second antibody, all $\mathrm{mAbs}$ screened by the procedure were IgG classes. Of the 79 clones, 23 were eventually chosen for further study; the immunoreactivities of some representatives are shown in Fig. 2A. To determine whether these mAbs could inhibit PLP phosphatase activity, samples of the enzyme were incubated with $\mathrm{mAbs}$, which had been purified by protein-A Sepharose affinity column. Of four representative mAbs tested, one (hPLPP1) mAb inhibited enzyme activity by a maximum of $43 \%$, the others (hPLPP2, hPLPP3, and hPLPP4) showed no inhibitory effect (Fig. 2B). This inhibitory effect of hPLPP1 may have been due to its binding at or near the active site, or to the immobilization of the enzyme due to hPLPP1 binding to epitopes remote from the active site. Another possibility is that antibody binding caused inactivation by inducing conformational changes. These potential conformation changes induced by hPLPP1 binding require further investigation. 
(A)

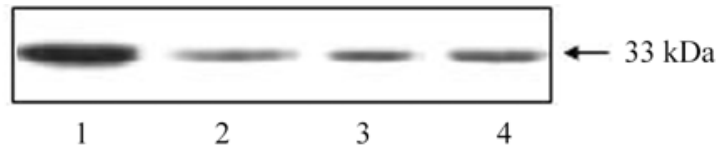

(B)

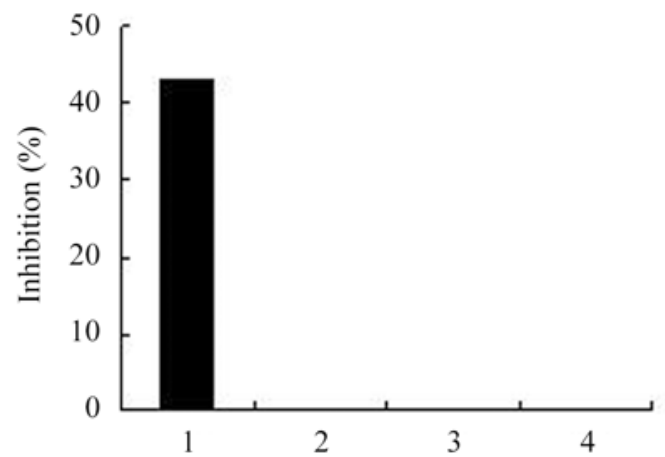

Fig. 2. The corresponding immunoblots of purified human PLP phosphatase probed with representative mAbs (A) and inhibition of PLP phosphatase activity by the purified mAbs (B): lane 1, hPLPP1; lane 2, hPLPP2; lane 3, hPLPP3; lane 4, hPLPP4. All $\mathrm{mAbs}$ specifically recognized a protein band of $33 \mathrm{kDa}$. Purified human PLP phosphatase $(1 \mu \mathrm{g}$ in $10 \mu \mathrm{l}$ of $0.1 \mathrm{M}$ potassium phosphate, $\mathrm{pH}$ 7.2) was incubated with purified mAbs $(10 \mu \mathrm{g}$ in $90 \mu \mathrm{l}$ PBS) for $1 \mathrm{~h}$ at room temperature. Sample enzyme activities were determined as described in Materials and Methods. Fresh culture supernatant was used as a control.

To examine the reactivities of these mAbs, we digested purified enzyme with V-8 protease and immunoblotted digested samples. Of the mAbs tested, two subgroups recognizing different peptide fragments were identified (Fig. 3). hPLPP1 produced a band pattern that differed from the others (hPLPP2, hPLPP3, and hPLPP4). Moreover, mAbs hPLPP2, 3, and 4 produced similar band patterns indicating that they recognized similar epitopes. The results of our immunoreactivity analysis support the possibility that human PLP phosphatase differs from the other mammal PLP phosphatases either in terms of amino acid sequence or protein structure. Although PLP phosphatase is known to show high sequence homology among species, we believe that human and other animal PLP phosphatases differ in terms of their tertiary or quaternary structures.

Protein-protein interactions of anti-PLP phosphatase monoclonal antibodies with PLP phosphatase To quantitatively

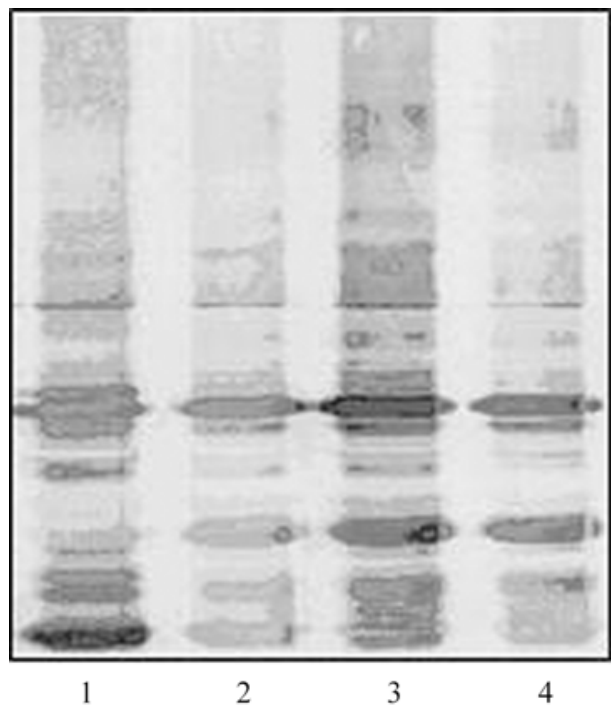

Fig. 3. Immunoreactivities of different $m A$ bs with purified human PLP phosphatase that had been digested with Staphylococcus aureus V-8 protease and separated by SDS-PAGE. Lanes are as follows: lane 1, hPLPP1; lane 2, hPLPP2; lane 3, hPLPP3; lane 4, hPLPP4.

compare the antigenic reactivities of anti-PLP phosphatase monoclonal antibodies with PLP phosphatase, we examined interactions between PLP phosphatase and its mAbs directly in Pharmacia BIAcore. The equilibrium binding antibody can be measured in a variety of ways, as long as the complex can be separated from free ligand once the reaction has reached equilibrium. However, few methods allow an analysis of interactions in real time, and thus the determination of kinetic rate constants. Biosensor technology uses the phenomenon of surface plasmon resonance to monitor the interaction between an immobilized ligand and a protein in a flowing system (Fagerstam et al., 1992; Malmqvist, 1993). By using the methods described above, $k_{\text {on }}$ and $k_{\text {off }}$ values were calculated for PLP phosphatase mAbs. Each measurement was performed in duplicate up to four times on different surfaces. The results of these kinetic experiments are summarized in Table 1. In particular, the binding affinities of all four antiPLP phosphatase mAbs for PLP phosphatase were similar. All $\mathrm{mAbs}$ showed had very slow dissociation, close to the limits of the machine $\left(\sim 1 \times 10^{-5} \mathrm{~s}^{-1}\right)$, and no differences were observed between their dissociation rate constants $\left(k_{\text {off }}\right)$. These results indicate that the molecular recognition processes of

Table 1. Interaction between anti-PLP phosphatase monoclonal antibody and PLP phosphatase

\begin{tabular}{cccc}
\hline mAbs & $\begin{array}{c}\text { Association rate constant } \\
\left(k_{\mathrm{on}}\right)\left(\mathrm{M}^{-1} \mathrm{~s}^{-1}\right)\end{array}$ & $\begin{array}{c}\text { Dissociation rate constant } \\
\left(k_{\mathrm{off}}\right)\left(\mathrm{s}^{-1}\right)\end{array}$ & $\begin{array}{c}\text { Equilibrium dissociation } \\
\text { constant }\left(K_{\mathrm{D}}\right)(\mathrm{nM})\end{array}$ \\
\hline hPLPP1 & $5.0 \times 10^{4}$ & $5.0 \times 10^{-5}$ & $1.0 \pm 0.12$ \\
hPLPP2 & $3.8 \times 10^{4}$ & $5.2 \times 10^{-5}$ & $1.3 \pm 0.26$ \\
hPLPP3 & $3.9 \times 10^{4}$ & $5.1 \times 10^{-5}$ & $1.3 \pm 0.18$ \\
hPLPP4 & $4.7 \times 10^{4}$ & $5.2 \times 10^{-5}$ & $1.1 \pm 0.32$ \\
\hline
\end{tabular}

Results are the averages of two separate experiments, and errors represent the ranges of the two data sets. 
(A)

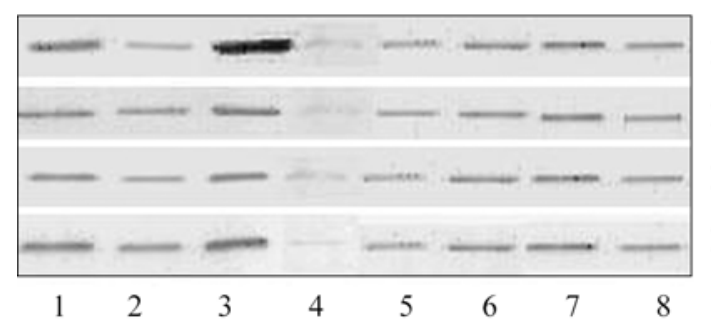

(B)

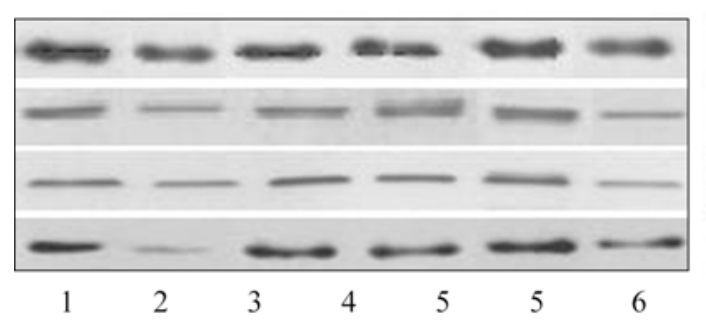

hPLPP 1

hPLPP 2

hPLPP 3

hPLPP 4

(C)

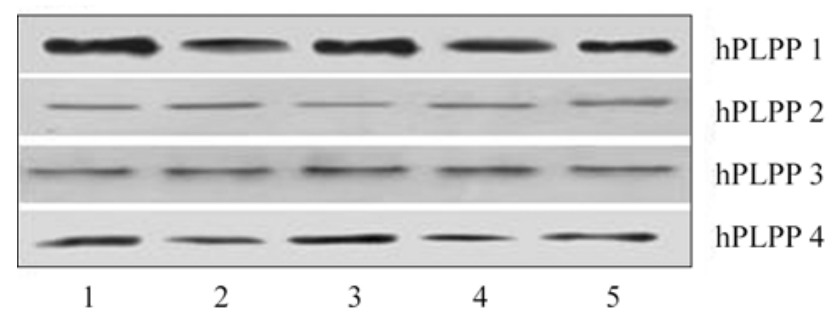

Fig. 4. Cross-reactivites of PLP phosphatase from mammalian and avian species (A), various rat tissues (B), and mammal cell lines (C) to mAbs against human PLP phosphatase. (A) Lane 1, human; lane 2, bovine; lane 3, rat; lane 4, chicken; lane 5, rabbit; lane 6 , cat; lane 7, dog; lane 8 , porcine. All animal brains were removed, and total proteins of homogenates were immunoblotted with PLP phosphatase mAbs. (B) Lane 1, brain; lane 2, heart; lane 3, lung; lane 4, liver; lane 5, stomach; lane 6, muscle. (C) Lane 1, PC12; lane 2, J774A1; lane 3, HepG2; lane 4, SiHa; lane5, HL3T1. Total proteins were extracted and immunoblotted with hPLPP mAbs.

each $\mathrm{mAb}$ with respect to PLP phosphatase have almost identical kinetic parameters.

\section{Cross-reactivity of mAbs with human PLP phosphatases} from other mammals and in different human cell lines In order to examine the cross-reactivities of the four Abs, we isolated human PLP phosphatase from human, bovine, rat, chicken, rabbit, cat, dog, and porcine brains. Total proteins in brain homogenates were separated by SDS-PAGE, transferred to nitrocellulose membranes, and probed with the four human PLP phosphatase mAbs. Immunoreactive bands on Western blot appeared as a strong single protein band at $33 \mathrm{kDa}$ for all animals tested (Fig. 4A). However, the intensity of the crossreactivity band for chicken was weak compared to those of the mammals. This result suggests that mammalian brain PLP phosphatases share a common epitope and a similar protein structure, while avian brain PLP phosphatase is immunologically distinct from mammalian brain PLP phosphatases, at least on one epitope.

We also examined the immunoreactivity of anti-human PLP phosphatase mAbs for PLP phosphatase from various rat tissues, namely, brain, heart, lung, liver, stomach, and muscle. As shown in Fig. 4B, anti-human PLP phosphatase mAbs strongly recognized the same protein band on immunoblots of the total proteins of various rat tissues. However, PLP phosphatase bands were somewhat weaker in heart and muscle than in other tissues. These results indicate either that PLP expression is differentially regulated in tissues, or that there are structural differences between the PLP phosphatase enzymes found in various tissues.

Next, we determined the immunoreactivity of PLP phosphatase in several mammal cell lines, i.e., mouse neuronal cells (PC12), mouse macrophages (J774A1), human hepatocellular carcinoma cells (HepG2), human squamous carcinoma cells (SiHa), and human cervical adenocarcinoma cells (HL3T1), using PLP phosphatase mAbs. Although mAbs strongly recognized a PLP phosphatase protein band in all of these cell lines examined, the immunoreactive bands of the same molecular mass in other mammal cell lines tested showed almost identical patterns.

Recently, we showed that PLP phosphatase is differentially expressed in a tissue-specific manner in man (Jang et al., 2003). Based on the levels of its mRNA expression, the widespread distribution of PLP phosphatase in human tissue is consistent with its essential role in cellular metabolism. In the present study, Western blot analysis showed that PLP phosphatase is most highly expressed in liver, testes, and in all regions of the central nerve system except the spinal cord, whereas its expression was low in the pancreas and heart. These results concur with its tissue-specific mRNA expression. In this regard, it should be noted that pyridoxal kinase is expressed in all organs (Hanna et al., 1997), whereas PNP oxidase is selectively expressed in liver and kidney (Buss et al., 1980).

In conclusion, this study describes the production and characterization of the first complete set of high-affinity mAbs against human PLP phosphatase. Since antibodies enable the specific and sensitive detection of enzymes in tissues, these $\mathrm{mAbs}$ could be used to quantitatively evaluate protein expression levels, and should substantially benefit immunohistochemical studies on the distribution of PLP phosphatase in the brain. Furthermore, human PLP phosphatase mAbs could provide us with detection tools for monitoring and diagnosing vitamin $\mathrm{B}_{6}$-related disorders.

Acknowledgments This work was supported by a $21^{\text {st }}$ Century Brain Frontier Research Grant (M103KV010019-03K220101910) and Basic Research Program (R01-2005-000-10004-0) from the Korean Ministry of Science and the Korean Engineering Foundation, and in part by a Regional Research Center (RRC) Grant from the Korean Ministry of Commerce, Industry, and Energy, and by a Research Grant from Hallym University. 


\section{References}

Anderson, B. B., Newmark, P. A., Rawlins, M. and Green, R. (1974) Plasma binding of vitamin $B_{6}$ compounds. Nature 250, 502-504.

Bull, H., Murray, P. G., Thomas, A. M. and Nelson, P. N. (2002) Acid phosphatases. Mol. Pathol. 55, 65-72.

Buss, D. D., Hamm, M. W., Mehansho, H. and Henderson, L. M. (1980) Transport and metabolism of pyridoxine in the perfused small intestine and the hind limb of the rat. J. Nutr. 110, 16551663.

Choi, E. Y., Park, S. Y., Jang, S. H., Song, M. S., Cho, S. W. and Choi, S. Y. (1995) Production and characterization of monoclonal antibodies to bovine brain succinic semialdehyde reductase. J. Neurochem. 64, 371-377.

Choi, E. Y., Jang, S. H. and Choi, S. Y. (1996) Human brain GABA transaminase is immunologically distinct from those of other mammalian brain. Neurochem. Int. 28, 597-600.

Eum, W. S., Choi, H. S., Kim, D. W., Jang, S. H., Choi, S. H., Kim, S. Y., Park, J., Kang, J. H., Cho, S. W., Kwon, O. S., Hwang, I. K., Yoo, K. Y., Kang, T. C., Won, M. H. and Choi, S. Y. (2005) Production and characterization of monoclonal antibodies against human ceruloplasmin. J. Biochem. Mol. Biol. 38, 71-76.

Fagerstam, L. G., Karlsson, A. F., Karlsson, R., Persson, B. and Ronnberg, I. (1992) Biospecific interaction analysis using surface plasmon resonance detection applied to kinetic, binding site and concentration analysis. J. Chromatogr. 597, 397-410.

Fonda, M. L. (1992) Purification and characterization of vitamin $\mathrm{B}_{6}$-phosphate phosphatase from human erythrocytes. J. Biol. Chem. 267, 15978-15983.

Fonda, M. L. and Zhang, Y. (1995) Kinetic mechanism and divalent metal activation of human erythrocyte pyridoxal phosphatase. Arch. Biochem. Biophys. 320, 345-352.

Gao, G. and Fonda, M. L. (1994a) Kinetic analysis and chemical modification of vitamin $\mathrm{B}_{6}$ phosphatase from human erythrocytes. J. Biol. Chem. 269, 7163-7168.

Gao, G. and Fonda, M. L. (1994b) Identification of an essential cysteine residue in pyridoxal phosphatase from human erythrocytes. J. Biol. Chem. 269, 8234-8239.

Gao, G. and Fonda, M. L. (1994c) Evidence for a phosphoenzyme intermediate formed during catalysis by pyridoxal phosphatase from human erythrocytes. Arch Biochem. Biophys. 313, 166172.

Glenn, G. M., Krober, M. S., Kelly, P., McCarty, J. and Weir, M. (1995) Pyridoxine as therapy in theophyline-induced seizure. Ver. Hum. Nerol. 395, 177-194.

Gospe Jr, S. M., Olin, K. L. and Keen, C. L. (1994) Reduced GABA synthesis in pyridoxine dependent seizures. Lancet 343, 1133-1134.

Hanna, M. C., Turner, A. J. and Kirkness, E. F. (1997) Human pyridoxal kinase. J. Biol. Chem. 272, 10756-10760.

Harris, H. (1990) The human alkaline phosphatases: what we know and what we don't know. Clin. Chim. Acta 186, 133150 .
Jang, Y. M., Kim, D. W., Kang, T. C., Won, M. H., Baek, N. I., Moon, B. J., Choi, S. Y. and Kwon, O. S. (2003) Human pyridoxal phosphatase: Molecular cloning, functional expression, and tissue distribution. J. Biol. Chem. 278, 5004050046.

Kwok, F. and Churchich, J. E. (1992) Pyridoxine-5'-P oxidase; in Chemistry and Biochemistry of Flavoenzymes, Muller, F. (ed.) pp. 1-20, CRC Press, London, UK.

Kwon, O. S., Kwok, F. and Churchich, J. E. (1991) Catalytic and regulatory properties of native and chymotrypsin-treated pyridoxine-5-phosphate oxidase. J. Biol. Chem. 266, 2213622140.

Laemmli, U. K. (1970) Cleavage of structural proteins during the assembly of the head of bacteriophage T4. Nature 227, 680685.

Lee, H. Y., Eum, W. S., Kim, D. W., Lee, B. R., Yoon, C. S., Jang, S. H., Choi, H. S., Choi, S. H., Back, N. I., Kang, J. H., Kang, T. C., Won, M. H., Cho, S. W., Lee, K. S., Park, J. and Choi, S. Y. (2003) Isolation and identification of antioxidant enzyme catalase stimulatory compound from Garnoderma lucidum. J. Biochem. Mol. Biol. 36, 450-455.

Li, T. K., Lumeng, L. and Veitch, R. L. (1974) Regulation of pyridoxal-5'-phosphate metabolism in liver. Biochem. Biophys. Res. Commun. 61, 677-684.

Lumeng, L. and Li, T. K. (1975) Characterization of the pyridoxal5'-phosphate and pyridoxamine-5'-phosphate hydrolase activity in rat liver: Identity with alkaline phosphatase. J. Biol. Chem. 250, 8126-8131.

Malmqvist, M. (1993) Biospecific interaction analysis using biosensor technology. Nature 361, 186-187.

McCormick, D. B., Gregory, M. E. and Snell, E. E. (1961) Pyridoxal phosphokinase I: assay, distribution, purification and properties. J. Biol. Chem. 236, 2076-2084.

Meister, A. (1990) On the transamination of enzymes. Ann. N. Y. Acad. Sci. 585, 13-31.

Ngo, E. O., LePage, G. R., Thanassi, J. W., Meisler, N. and Netter, L. M. (1998) Absence of pyridoxine-5'-phosphate oxidase (PNPO) activity in neoplastic cells: isolation, characterization, and expression of PNPO cDNA. Biochemistry 37, 7741-7748.

Snell, E. E. (1990) Vitamine $B_{6}$ and decarboxylation of histidine. Ann. N. Y. Acad. Sci. 585, 1-12.

Snell, E. E. and Haskell, B. E. (1971) The metabolism of vitamine $\mathrm{B}_{6}$; in Comprehensive Biochemistry, Florkin, M., and Stotz, E. H., (eds.), pp. 47-71, Elsevier Scientific Publishing Co., Netherlands.

Towbin, H., Staehelin, T. and Gordon, J. (1978) Electrophoretic transfer of proteins from polyacrylamide gels to nitrocellulose sheets: procedures and some applications. Proc. Natl. Acad. Sci. USA 76, 7350-4354.

Waymire, K. G., Mahuren, J. D., Jaje, J. M., Guilarte, T. R., Coburn, S. P. and MacGregor, G. R. (1995) Mice lacking tissues non-specific alkaline phosphatase die from seizures due to defective metabolism of vitamin $\mathrm{B}_{6}$. Nat. Genet. 11, 45-51. 